Our thanks to all those who have helped with this issue of Bioanalysis. Listed below are authors, referees and others who have kindly given their time, effort and expertise; their generosity has helped establish this publication.

$\begin{array}{llll}\text { Abdul B } & \text { Geist B } & \text { Mulder H } & \text { Tichy A } \\ \text { Ahene A } & \text { Gouty D } & \text { Murray T } & \text { Timmerman P } \\ \text { Barth T } & \text { Gross E } & \text { O'Hara D } & \text { Trivedi V } \\ \text { Bhushan R } & \text { Hayes M } & \text { Peaper D } & \text { Upadhyay V } \\ \text { Bonato P } & \text { Henderson N } & \text { Ploch S } & \text { van Amsterdam P } \\ \text { Bracewell D } & \text { Hodsdon M } & \text { Rahavendran R } & \text { Verch T } \\ \text { Bridle H } & \text { Huang N } & \text { Ruterbories K } & \text { Weiner R } \\ \text { Brundy-Kloeppel M } & \text { Inglese B } & \text { Saad O } & \text { White J } \\ \text { Campagna S } & \text { Jarvis M } & \text { Sanyal M } & \text { Wickremsinhe E } \\ \text { Carrasco-Triguero M } & \text { Johnson M } & \text { Shah G } & \text { Woolley C } \\ \text { Chen Y } & \text { Kagel J } & \text { Shankar G } & \text { Wright P } \\ \text { Cheng K } & \text { Kaur S } & \text { Shi Y } & \text { Xu K } \\ \text { Clark T } & \text { Knotts K } & \text { Shrivastav P } & \text { Yadav M } \\ \text { Companjen A } & \text { Kulkarni M } & \text { Siluk D } & \text { Yamini Y } \\ \text { Cosentino G } & \text { Lai R } & \text { Simoes R } & \text { Yang T } \\ \text { Couchman L } & \text { Li W } & \text { Smales C } & \text { Zhong Z } \\ \text { Dere R } & \text { Liu K } & \text { Smeraglia J } & \text { Zimmer J } \\ \text { Desai D } & \text { Manro J } & \text { Staack R } & \\ \text { Dong Y } & \text { Mess J } & \text { Tang Y } & \\ \text { Egan A } & \text { Mills G } & \text { Tasso L } & \end{array}$

\title{
INDEX OF ADVERTISERS
}

\section{Company}

Publish your research with Bioanalysis

Inside Front Cover

Bioanalysis zone - get in the zone

Advertise with Bioanalysis

Subscribe to Bioanalysis

Bioanalysis zone - have your say

Bioanalysis trial

Pharmaceutical Patent Analyst 